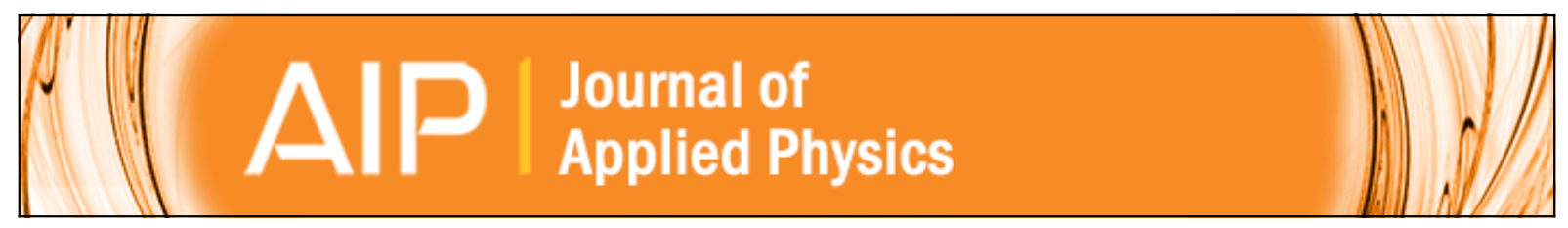

\title{
Exciton capture by nanocrystals in a polymer matrix
}

A. Shik, G. Konstantatos, E. H. Sargent, and H. E. Ruda

Citation: Journal of Applied Physics 94, 4066 (2003); doi: 10.1063/1.1599974

View online: http://dx.doi.org/10.1063/1.1599974

View Table of Contents: http://scitation.aip.org/content/aip/journal/jap/94/6?ver=pdfcov

Published by the AIP Publishing

\section{Articles you may be interested in}

CdS nanocrystal-sensitized solar cells with polyaniline as counter electrode

J. Renewable Sustainable Energy 4, 043108 (2012); 10.1063/1.4737133

Excitons in Si $1-x$ Ge x nanocrystals: Ab initio calculations

J. Appl. Phys. 103, 103716 (2008); 10.1063/1.2913314

Effects of molecular interface modification in hybrid organic-inorganic photovoltaic cells

J. Appl. Phys. 101, 114503 (2007); 10.1063/1.2737977

Exciton relaxation processes in colloidal core/shell ZnSe/ZnS nanocrystals

Appl. Phys. Lett. 82, 418 (2003); 10.1063/1.1537050

Comparison of density matrix renormalization group calculations with electron-hole models of exciton binding in conjugated polymers

J. Chem. Phys. 108, 7451 (1998); 10.1063/1.476165

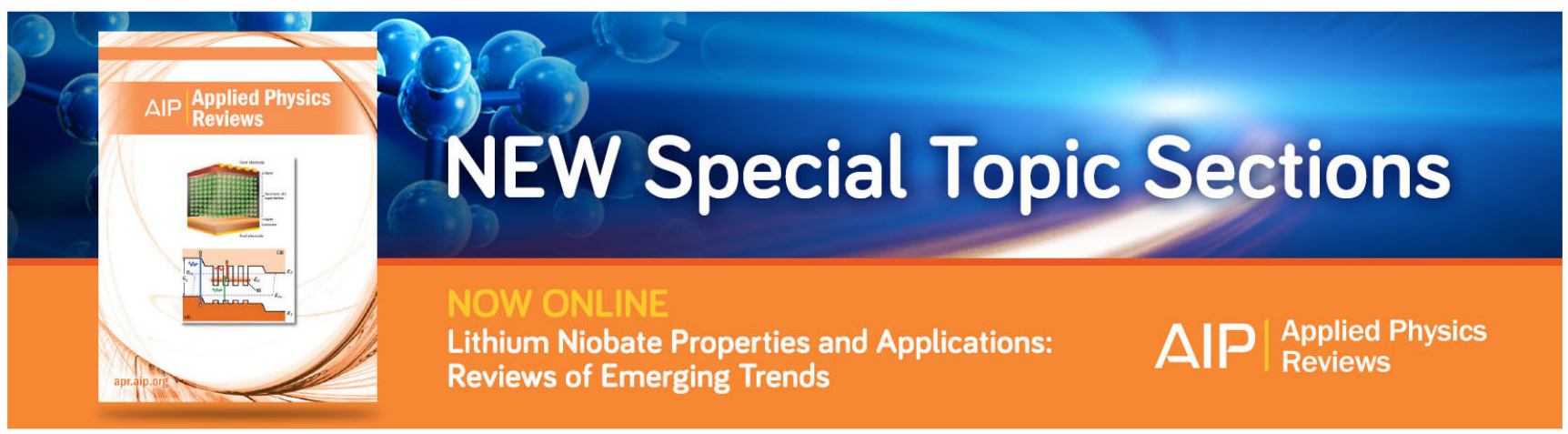




\title{
Exciton capture by nanocrystals in a polymer matrix
}

\author{
A. Shik ${ }^{\mathrm{a})}$ \\ Electronic and Photonic Material Group, University of Toronto, Toronto M5S 3E4, Canada \\ G. Konstantatos and E. H. Sargent \\ Department of Electrical and Computer Engineering, University of Toronto, Toronto M5S 3G4, Canada \\ H. E. Ruda \\ Electronic and Photonic Material Group, University of Toronto, Toronto M5S 3E4, Canada
}

(Received 12 May 2003; accepted 13 June 2003)

\begin{abstract}
A model for the capture of nonequilibrium excitons by semiconductor nanocrystals in a polymer matrix via dipole-dipole interactions is developed. The transition probability $W$ is found by calculating Joule energy losses in the nanocrystal induced by an external oscillating dipole. To find the net capture rate, the problem is solved simultaneously with the diffusion equation describing generation and recombination of excitons in the polymer matrix and their transport to the nanocrystal interface. In real conducting polymers the capture rate is often limited not by dipole interactions but by diffusion transport so that the key condition for high efficiency of light-emitting devices based on organic-inorganic nanocomposites is that the diffusion length of excitons in the polymer matrix exceeds the average internanocrystal spacing. (C) 2003 American Institute of Physics. [DOI: 10.1063/1.1599974]
\end{abstract}

\section{INTRODUCTION}

Capture of nonequilibrium carriers by nanocrystals (NCs) which plays a decisive role in NC-based light-emitting structures, is usually described in terms of multiphonon separate capture of electrons and holes. This approach fails in the polymer-nanocrystal composites widely used in recent years for photonic devices. ${ }^{1}$ Excitations created by optical or electrical pumping in the polymer matrix exist, as a rule, in the form of Frenkel excitons, rather than free electron-hole pairs and one of the most important mechanisms of carrier capture by a NC is the Förster one, which is the energy transfer from one system (in our case the Frenkel exciton in a polymer) to another (electron-hole pair in a NC) via dipole-dipole excitation. An alternative mechanism-separate capture of electron and holes-may be much weaker due to rather large binding energy of Frenkel excitons, typically $0.2-0.4 \mathrm{eV}^{2}$ The Förster capture may be of a special importance if we take into account that in most polymer-based composites, embedded NCs are passivated by ligand molecules creating an additional shell, which inhibits direct capture of carriers. The present communication is devoted to a theoretical description of the capture of nonequilibrium Frenkel excitons created in a polymer matrix by semiconductor NCs-the process which ultimately determines the quantum yield of polymer-semiconductor light-emitting devices.

\section{TRANSITION PROBABILITY FOR A SINGLE NANOCRYSTAL}

We consider a spherical semiconductor NC with radius $a$ and a Frenkel exciton at the point $\mathbf{r}_{e}\left(\left|\mathbf{r}_{e}\right|>a\right)$ in a polymer

\footnotetext{
a) Author to whom correspondence should be addressed; electronic mail: shik@ecf.utoronto.ca
}

matrix and calculate the probability of exciton recombination with simultaneous generation of an electron-hole pair (or a Wannier-Mott exciton) in a NC via electrostatic interaction. Our theoretical approach is similar to that developed in Refs. 3 and 4 for the reciprocal problem of energy transfer from a semiconductor quantum well or dot to a polymer matrix. The corresponding probability can be expressed in terms of classical Joule losses

$$
W=\frac{1}{2 \pi \hbar} \int \operatorname{Im} \epsilon(\mathbf{r}, \omega)|\mathbf{E}(\mathbf{r})|^{2} d^{3} \mathbf{r} .
$$

Here $\mathbf{E}(\mathbf{r})$ is the electric field of an external dipole with the frequency $\omega$ in the medium to which energy is to be transferred, $\epsilon(\mathbf{r}, \omega)$ is the dielectric function of this medium and integral is taken over its volume. In spite of the seemingly classical character of Eq. (1), it is an exact expression if $\epsilon(\mathbf{r}, \omega)$ is taken from strict quantum mechanical calculations considering all possible electron transitions at the frequency $\omega$ and is equivalent to calculations of the corresponding transition probabilities (see Refs. 3 and 4). In cited articles devoted to energy losses by quantum wells or dots, the role of the energy recipient medium was played by the matrix so that $\epsilon(\mathbf{r}, \omega)$ refers to the polymer and the integration covered the whole matrix. In our case, energy is transferred to the NC and the integration region is the sphere $r<a$. The situation is quite opposite to that of Refs. 3 and 4 and we are no longer interested in dielectric properties of a polymer. The energy recipient medium is now the $\mathrm{NC}$ so that the function $\epsilon(\mathbf{r}, \omega)$ is determined by its energy spectrum and electron and hole wave functions.

Generally speaking, the energy spectrum of an ideal NC is purely discrete and the frequency dispersion of $\operatorname{Im} \epsilon$ consists of a series of $\delta$ peaks. Since the Frenkel exciton is also characterized by a fixed discrete energy $E_{F}\left(\omega=E_{F} / \hbar\right)$, we 
can expect that the electron transition we are interested in can occur only at resonance when $E_{F}$ coincides with a gap between some electron and hole states or corresponding Wannier-Mott exciton energies in NC. Such coincidence (assumed also in Ref. 5) occurs only occasionally, so that for most ideal NCs the Förster-type transitions are impossible. In other words, dependence of the transition probability on system parameters (e.g., on the NC radius $a$ ) should represent a system of $\delta$ peaks. However, in real systems such resonant behavior may not be observed. If the difference in energy gaps between a polymer and a semiconductor is large, the energy $E_{F}$ for a moderately sized NC will lie far above the ground state of an electron-hole pair (or Wannier-Mott exciton), close to the states with large quantum numbers. These states in ideal NCs are highly degenerate but any deviation from ideal spherical shape in real structures will remove this degeneracy creating instead a number of quasi-continuous levels. Furthermore, inevitable dispersion of NC size will additionally broaden any spectral dependence observed in a macroscopic sample. It is also worth adding that, due to the relatively low electron affinities in most conducting polymers, the band diagram of polymer-semiconductor interface often represents the so-called type-II heterostructure where $\mathrm{NC}$ is a potential well for electrons but a barrier for holes. ${ }^{1}$ In this case the hole spectrum in NCs is continuous and Förster transitions are always possible.

All of the mentioned arguments suggest that neglecting the detailed quantum structure of the energy spectrum in
NCs may result in a reasonable, at least qualitatively, description of the Förster mechanism in real structures with not extremely small NCs. For further analytical calculations we will use $\epsilon(\mathbf{r}, \omega)$ for bulk semiconductors and containing no coordinate dependence. For exact quantitative results one can use the dielectric functions obtained by numerical simulation $^{6}$ for particular quantum dots.

In the above-mentioned continuous approximation, Eq. (1) is simplified. Using the connection between $\operatorname{Im} \epsilon(\omega)$ and the optical absorption coefficient $\alpha(\omega)$, we have

$$
W=\frac{n_{r} c \alpha(\omega)}{2 \pi \hbar \omega} \int|\mathbf{E}(\mathbf{r})|^{2} d^{3} \mathbf{r}
$$

where $n_{r}$ is the NC refractive index. Thus for calculations of the Forster transition rate we need the spatial distribution of electric field created in the NC by a single Frenkel exciton in the immediate vicinity of it. To obtain a simple analytical formula, we ignore for the time being the difference in polarizability between the $\mathrm{NC}$ and the matrix, describing them by the same effective real dielectric constant $\epsilon_{0}$ (this approximation also implies $\operatorname{Im} \epsilon \ll \epsilon_{0}$ ). This allows us to ignore the image forces and to write for a dipole $\mathbf{d}$ at the origin, the squared electric field in the point $\mathbf{R}$ as $E^{2}=(1$ $\left.+3 \cos ^{2} \Theta\right) d^{2} / \epsilon_{0}^{2} R^{6}$, where $\Theta$ is the angle between $\mathbf{d}$ and $\mathbf{R}$. If the dipole is located at the point with polar coordinates $\left(r_{e}, 0,0\right)$ and oriented along the polar direction $\left(\theta_{e}, 0\right)$, then at an arbitrary point $(r, \theta, \phi)$

$$
E^{2}\left(r, \theta, \phi ; r_{e}, \theta_{e}\right)=\frac{d^{2}\left\{r^{2}+r_{e}^{2}-2 r r_{e} \cos \theta+3\left[\cos \theta_{e}\left(r \cos \theta-r_{e}\right)+r \sin \theta_{e} \sin \theta \cos \phi\right]^{2}\right\}}{\varepsilon_{0}^{2}\left(r^{2}+r_{e}^{2}-2 r r_{e} \cos \theta\right)^{4}} .
$$

Substituting Eq. (3) into Eq. (2), integrating over the whole $\mathrm{NC}$ volume, and averaging over the dipole orientation $\theta_{e}$, we obtain the resulting formula for the transition probability

$$
W=\frac{4 n_{r} c \alpha(\omega) d^{2} a^{3}}{3 \hbar \omega \varepsilon_{0}^{2}\left(r_{e}-a\right)^{3}\left(r_{e}+a\right)^{3}} .
$$

To estimate $W$ given by Eq. (4), we assume $d=1 \mathrm{D}$ unit ( $\left.10^{-18} \mathrm{CGSE}\right), n_{r}=3, \varepsilon_{0}=5, \alpha=10^{5} \mathrm{~cm}^{-1}$, and $\hbar \omega=2 \mathrm{eV}$. The value of $W$ increases dramatically when the position of the Frenkel exciton $r_{e}$ approaches the NC surface. For estimates, we assume a minimal possible value of $r_{e}-a$ to be of the order of interatomic distance in the polymer $a_{p}$. Taking $a_{p}=0.5 \mathrm{~nm}$, we obtain for the maximal transition probability $W \sim 1.5 \times 10^{11} \mathrm{~s}^{-1}$ (transition time $\sim 7 \mathrm{ps}$ ). Note that in the limit $r_{e}-a \ll a$, when $W$ is maximal, the answer does not depend on the NC size $a$ and is inversely proportional to the cube of distance between the exciton and NC surface: $W$ $\sim\left(r_{e}-a\right)^{-3}$. This asymptotic answer could be also obtained by replacing NC with an infinite semispace, which is possible due to the very fast decrease of $E^{2}$ with distance.

The formula Eq. (3) ignores modification of the dipole potential by the difference of polymer and semiconductor dielectric constants, $\varepsilon_{1}$ and $\varepsilon_{2}$. In our system with a spherical dielectric nonuniformity this modification consists in theappearance of image charges so that the potential of a charge or a dipole is given by an infinite series. ${ }^{7}$ The situation is, however, simplified for the case of NCs noticeably larger than $a_{p}$. In this case, the NC interface can be approximated by a plane and the potential of a dipole beyond the interface coincides with that in uniform medium with the dielectric constant $\left(\varepsilon_{1}+\varepsilon_{2}\right) / 2$. Thus the capture probability is described, as before, by Eq. (4) with the replacement $\epsilon_{0} \rightarrow\left(\epsilon_{1}\right.$ $\left.+\varepsilon_{2}\right) / 2$.

\section{NET CAPTURE RATE}

The knowledge of the transition probability Eq. (4) and its dependence on exciton distance from the $\mathrm{NC}$ center $W\left(r_{e}\right)$ allows us to find the net rate of carrier capture by NC. A complete description of the process must include generation of excitons in the polymer, their diffusion towards NCs, partial recombination in the matrix, and capture by NCs. The specific feature of the Förster capture [Eq. (4)] is its very strong distance dependence so that at $r_{e}-a$ exceeding several $a_{p}, W$ becomes negligibly small. For this reason, we can 
analyze the processes in the polymer matrix considering NC capture as a phenomenon occurring almost directly at the $\mathrm{NC}$ interface and described by some effective surface recombination rate $s$. To obtain the expression for $s$, we note that if the exciton density in the vicinity of a NC is $n$, then the total rate of exciton capture is

$$
\frac{d n}{d t}=4 \pi n \int_{a+a_{p}}^{\infty} W\left(r_{e}\right) r_{e}^{2} d r_{e} \simeq \frac{\pi n n_{r} c \alpha(\omega) d^{2} a^{2}}{3 \hbar \omega \varepsilon_{0}^{2} a_{p}^{2}} .
$$

The recombination flux $j_{r}=\left(4 \pi a^{2}\right)^{-1}(d n / d t)$ and hence $s$, representing the proportionality factor between $j_{r}$ and $n$, is equal to

$$
s=\frac{n_{r} c \alpha(\omega) d^{2}}{12 \hbar \omega \varepsilon_{0}^{2} a_{p}^{2}}
$$

To describe the behavior of Frenkel excitons in the matrix, we find the radial distribution of their density $n(r)(r$ $>a$ is measured from the NC center) from the continuity equation

$$
D \frac{d}{r^{2} d r}\left(r^{2} \frac{d n}{d r}\right)=\frac{n}{\tau}-G
$$

where $G, D$, and $\tau$ are the generation rate, the diffusion co- efficient, and the lifetime of Frenkel excitons in a bulk polymer. One of the boundary conditions to Eq. (7) describes the capture

$$
D \frac{d n}{d r}(a)=\operatorname{sn}(a) .
$$

The other condition will be obtained by the approach similar to that used for description of nonequilibrium carriers in the low-temperature grown GaAs with arsenic clusters. ${ }^{8}$ We assume that NCs form a periodic lattice with unit cells centered by a NC. By symmetry, the diffusion flux must vanish at the cell boundaries. By analogy with the Wigner-Seitz method of band structure calculations, we replace the unit cell by a sphere with radius $R_{0}=[3 /(4 \pi N)]^{1 / 3}(N$ is the NC density), which results in the boundary condition

$$
\frac{d n}{d r}\left(R_{0}\right)=0
$$

With Eqs. (8) and (9) we find the distribution $n(r)$ and, particularly, the value of $n(a)$ determining the capture rate by a NC. We present the final expression for the quantum yield $\eta$ representing the fraction of all nonequilibrium excitons captured by $\mathrm{NCs}$

$$
\begin{aligned}
& \eta=\frac{4 \pi a^{2} \operatorname{sn}(a)}{\frac{4 \pi}{3}\left(R_{0}^{3}-a^{3}\right) G}=\frac{3 z}{y\left(x^{3}-1\right)} \cdot\left[\frac{(x y+1)(y-1) \exp [-y(x-1)]-(x y-1)(y+1) \exp [y(x-1)]}{(x y+1)(y-1-y z) \exp [-y(x-1)]-(x y-1)(y+1+y z) \exp [y(x-1)]}\right] \\
& L=\sqrt{D \tau}, \quad x=\frac{R_{0}}{a}, \quad y=\frac{a}{L}, \quad z=\frac{s L}{D}=s \sqrt{\frac{\tau}{D}}
\end{aligned}
$$

containing three dimensionless parameters: $x, y, z$. The first of them characterizes the $\mathrm{NC}$ density (the relative volume occupied by NCs is $4 \pi N a^{3} / 3 \equiv x^{-3}$ ), $y$ describes the properties of the matrix, while $z$ is proportional to $s$ and is the measure of the capture efficiency.

Let us discuss the requirements for these parameters securing high values of $\eta$. The question has a high applied importance since, due to a large quantum yield of radiative recombination in semiconductor NCs (considerably higher than in a polymer matrix), large $\eta$ is a condition of a noticeable increase of the light emitter efficiency in organicinorganic nanocomposites, as compared to that in a pure polymer. We note first of all that $\eta$ increasing with $z$, saturates at the value

$$
\eta_{\infty}=\frac{3}{y^{2}\left(x^{3}-1\right)}\left[\frac{(x y-1)(y+1) \exp [y(x-1)]-(x y+1)(y-1) \exp [-y(x-1)]}{(x y-1) \exp [y(x-1)]+(x y+1) \exp [-y(x-1)]}\right],
$$

which at large $x y=R_{0} / L$ is considerably less than 1 . This reflects the fact that at large $s$, the factor restricting NC capture is not the capture itself but the exciton diffusion to the NC surface and $\eta$ is determined by the interplay between diffusion and recombination in the matrix. To acquire high values of $\eta$, the sample must have not only effective Förster capture (large $s$ ) but also high enough diffusion length in the matrix, exceeding the NC separation. Quantitative estimates should answer two questions: (i) what values of $s$ are high enough to use the asymptotic expression Eq. (11) and (ii) what are the requirements for the polymer matrix guaranteering the corresponding values of $\eta_{\infty}$ to be close to unity.

We begin with the second question. Figure 1 demonstrates the values of $x$ and $y$ corresponding to $\eta_{\infty}=0.8$. To 


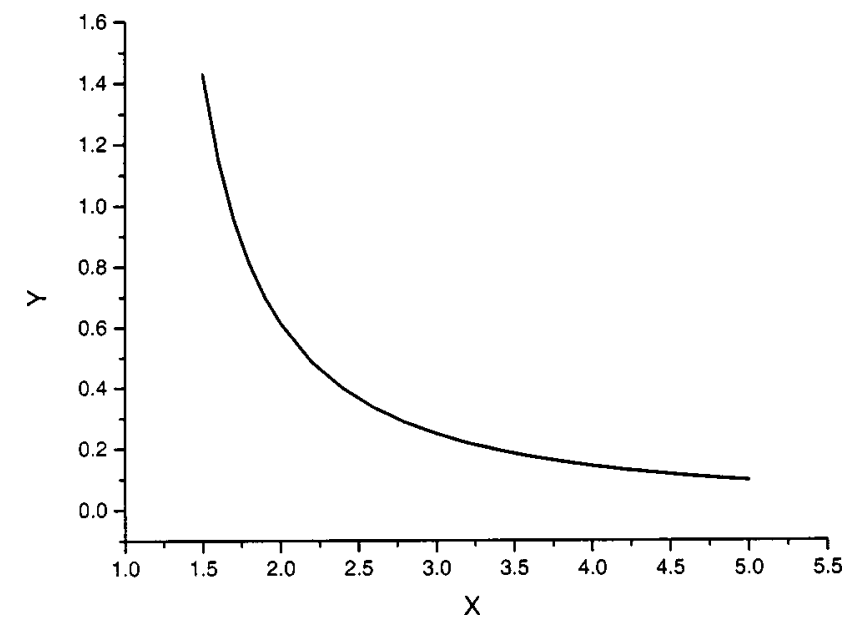

FIG. 1. Relationship between dimensionless nanocomposite parameters $x$ and $y$ corresponding to a capture efficiency $\eta_{\infty}=0.8$. The region below the curve corresponds to structures with higher efficiencies.

obtain higher $\eta_{\infty}$, the $x$ and $y$ values should lie below the curve. Since for a fixed $x$ (given NC density), $\eta_{\infty}$ is determined by one single parameter $y=a / L$, small NCs are preferable for reaching high $\eta$. We take for estimates the values $L \simeq 5 \mathrm{~nm}, D \simeq 2 \times 10^{-4} \mathrm{~cm}^{2} / \mathrm{s}$ experimentally determined for the PPV polymer. ${ }^{9}$ We see that for $x=2(12 \%$ volume of NCs), one can reach $\eta_{\infty}=0.8$ only for $a<3 \mathrm{~nm}$ while for $x$ $=5(1 \%$ volume of NCs) the estimate gives $a<0.5 \mathrm{~nm}$, which corresponds to a single interatomic distance and hence cannot be realized in a polymer with the given diffusion length $L$.

It can be easily shown from the general formula Eq. (10) that for the $x$ and $y$ corresponding to Fig. 1, $\eta_{\infty}$ approximates the real value $\eta$ with the $10 \%$ accuracy (in other words, $\eta \gtrsim 0.7$ ) if $z \gtrsim 10$ (at $x=2$ ) or $z \gtrsim 30$ (at $x=5$ ). For the $L$ and $D$ given above, it gives $s \gtrsim 10^{3} \mathrm{~cm} / \mathrm{s}$. Using the same values of parameters as in our previous estimates, we obtain from Eq. (6) values of $s$ several times larger. Thus, in most cases the main hurdle to high efficiency NC capture might be created not by weakness of dipole-dipole interaction but by low values of the exciton diffusion length in a polymer.

In this connection we point at a number of works where the values of $L$ in polymers noticeably exceeding $5 \mathrm{~nm}$ (used in our estimates) were observed. ${ }^{10-13}$ It allows us to look optimistically at the prospects of obtaining high quantum yield luminescence in organic-inorganic nanocomposites.

We emphasize once more that the capture probability Eq. (4) depends drastically on the minimal exciton NC distance $a_{p}$. Our estimate $a_{p} \sim 0.5 \mathrm{~nm}$ assumes direct polymer- nanocrystal contact while in many real composites NCs are protected by a layer of ligands suppressing surface recombination, which may increase effective $a_{p}$ up to $2-10 \mathrm{~nm}$ (depending on the length of ligand molecules) and suppress the capture efficiency. This agrees with the experimentally observed dependence of the electroluminescence intensity in $\mathrm{PbS} /$ polymer nanocomposites on the length of ligand chain ${ }^{14}$ and clearly displays the necessity of optimization the ligand material in light-emitting nanocomposites.

\section{CONCLUSION}

To summarize, we have considered capture of nonequilibrium excitons by semiconductor NCs in a polymer matrix via dipole-dipole (Förster) mechanism, which ultimately determines the efficiency of nanocomposite-based light emitters. The capture is a two-stage process including direct energy exchange between Frenkel excitons in a polymer and electron-hole pairs in NCs and diffusion delivery of excitons to the surface of NCs where this energy exchange is effective. Estimates show that in most real cases the bottleneck determining the net capture probability is related to the exciton diffusion. Thus high efficiency of capture and hence high quantum yield of light emission from NCs can be achieved by using polymers with high enough exciton diffusion length and minimizing the inter-NC separation, which, in turn, requires a suitably small size and high density of NCs.

${ }^{1}$ L. Bakueva, S. Musikhin, E. H. Sargent, H. E. Ruda, and A. Shik, in Handbook of Organic-Inorganic Hybrid Materials and Nanocomposites, edited by H. S. Nalwa (American Science, 2003).

${ }^{2}$ E. M. Conwell, Synth. Met. 83, 101 (1996).

${ }^{3}$ D. Basko, G. C. La Rocca, F. Bassani, and V. M. Agranovich, Eur. Phys. J. B 8, 353 (1999).

${ }^{4}$ D. M. Basko, V. M. Agranovich, F. Bassani, and G. C. La Rocca, Eur. Phys. J. B 13, 653 (2000).

${ }^{5}$ A. Engelmann, V. I. Yudson, and P. Reineker, Phys. Rev. B 57, 1784 (1998).

${ }^{6}$ L.-W. Wang and A. Zunger, Phys. Rev. B 53, 9579 (1996).

${ }^{7}$ V. V. Batygin and I. N. Toptygin, Problems in Electrodynamics (Academic, New York, 1978).

${ }^{8}$ H. Ruda and A. Shik, Phys. Rev. B 63, 085203 (2001).

${ }^{9}$ M. Pope and C. E. Swenberg, Electronic Processes in Organic Crystals and Polymers (Oxford University Press, Oxford, 1999), p. 852.

${ }^{10}$ T. Stübinger and W. Brütting, J. Appl. Phys. 90, 3632 (2001).

${ }^{11}$ A. Haugeneder et al., Phys. Rev. B 59, 15346 (1999).

${ }^{12}$ V. Choong, Y. Park, Y. Gao, T. Wehrmeister, K. Müllen, B. R. Hsieh, and C. W. Tang, Appl. Phys. Lett. 69, 1492 (1996).

${ }^{13}$ H. R. Kerp, H. Donker, R. B. M. Koehorst, T. J. Schaafsma, and E. E. van Faassen, Chem. Phys. Lett. 298, 302 (1998).

${ }^{14}$ L. Bakueva, S. Musikhin, M. A. Hines, T.-W. F. Chang, M. Tzolov, G. D. Scholes, and E. H. Sargent, Appl. Phys. Lett. 82, 2895 (2003). 